\title{
Retinal vascular architecture is maintained in retinal degeneration: corrosion cast and electron microscope study
}

\section{Abstract}

Purpose To demonstrate the changes of the retinal vascular architecture in the diffusely degenerated thin retina.

Methods Three-week-old weanling Wistar Kyoto rats were divided randomly into two groups. One group $(n=20)$ was fed a vitamin E-deficient solid diet and the other group $(n=20)$ was fed a solid rat chow diet. Rats were maintained on their respective diets for 14 months and then killed for scanning electron microscopy of vascular corrosion casts, light and electron microscopy and biochemical determinations.

Results The serum level of vitamin $\mathrm{E}$ in the E-deficient rats was $1.0 \pm 0.49 \mu \mathrm{g} / \mathrm{ml}$, while that in the rats fed a normal diet was $13.7 \pm 1.0 \mu \mathrm{g} / \mathrm{ml}$ (Student's $t$-test, $p=0.0001$ ). In vitamin E-deficient rats, light microscopy showed degenerated retinas only half as thick as normal. Corrosion casts and scanning electron microscopy revealed that the retinal capillaries of the entire retina were decreased in number and scattered with localised narrowing, calibre irregularity and frequent loop formation. In the posterior pole of the retina, some capillaries clustered into small tortuous knots. However, the two-layered architecture of the capillary network in the retina was maintained. The differences in calibre of retinal capillaries between the vitamin E-deficient and normal rats were statistically significant $(p<0.0001)$. No remarkable abnormal changes were observed in the large retinal vessels other than arterial calibre differences $(p<0.022)$. No arteriovenous shunts, crossing defects or microaneurysms were seen. Transmission electron microscopy revealed complete disappearance of the photoreceptor outer and inner segments and nuclei. The retinal pigment epithelium contained lipofuscin granules and retinal capillaries with narrow lumens. The capillary endothelial cells were thickened and had scarce cytoplasmic components with vacuoles and irregularly thickened basement membranes. The capillary pericytes had vacuoles. No abnormalities were seen in the control normal rats.

Conclusion These findings indicate that the decrease in retinal capillaries in vitamin Edeficient rats is secondary to retinal degeneration. It was assumed that the morphological changes in the capillary network reflected structural damage to the retinal vascular cells caused by free radicals and lipid peroxides generated by oxidation. However, even in such severe degeneration the retinal vascular architecture, including the main artery and vein and two-layer capillary networks, was maintained. This is may be because of the basic anatomical arrangement of the blood vessels.

Key words Corrosion cast, Electron microscopy, Rat, Retinal degeneration, Retinal vessels, Vitamin E deficiency

The retina has a beautiful vascular architecture composed of two layers of capillary networks. This architecture is easily demonstrated by corrosion casts and scanning electron microscopy. ${ }^{1,2}$ We are interested in what happens to the retinal vascular architecture in retinal diseases. The changes in the vascular network of the retina must be different in local and global lesions in the retina. Between the outer plexiform layer and the nerve fibre layer the sensory retina has vessels which form the two-layer networks of capillaries.

The purpose of the present study was to demonstrate the changes of retinal vascular architecture throughout the thinned degenerated retina. We used vitamin E-deficient rats for this purpose, because these rats have prominent retinal and rectus muscle capillary changes as well as severe changes in the neural retinal cells, ${ }^{3}$ and their retinas become thin. ${ }^{4}$
I.A. Bhutto
T. Amemiya
Department of
Ophthalmology
Nagasaki University School
of Medicine
Nagasaki 852-8501, Japan
Imran A. Bhutto, PhD
Department of
Ophthalmology
Nagasaki University School
of Medicine
1-7-1 Sakamoto
Nagasaki $852-8501$, Japan
Tel: +81 958497345
Fax: +81 958497347
e-mail:

This work was presented at the XIV International

Congress of Eye Research, Santa Fe, New Mexico, USA October 2000

Received: 31 October 2000 Accepted in revised form: 6 March 2001 


\section{Materials and methods}

\section{Experimental animals and diet}

Three-week-old weanling Wistar Kyoto (WKy) rats of both sexes weighing 40-50 g each were divided randomly into two groups. One group of 20 animals was fed a commercially prepared vitamin E-deficient solid diet obtained from Nippon Clea (Tokyo, Japan) for 14 months. The composition of the vitamin E-deficient diet is shown in Table 1. Another group of 20 control animals was maintained on a solid rat chow diet containing $5 \mathrm{mg}$ of vitamin E per $100 \mathrm{~g}$ of diet and all other nutrients (obtained from Oriental Yeast, Tokyo, Japan) for 14 months. The diets were stored at $4{ }^{\circ} \mathrm{C}$ until use. Distilled and deionised drinking water and diets were provided ad libitum.

All rats were housed individually in translucent plastic cages and maintained at a constant temperature of $21^{\circ} \mathrm{C}$ with $12 \mathrm{~h}$ light and dark cycles (light period, 07:00-19:00 hours) under illumination of about 25 footcandles from cool-white fluorescent tubelights (General Electric) at the Laboratory Animal Center for Biomedical Research, Nagasaki University School of Medicine. The animals were treated according to the ARVO Resolution on the Use of Animals in Research.

\section{Measurement of blood pressure and vitamin E serum level}

At regular intervals the rats were weighed, and their systolic blood pressure was measured by the tail-pulse pickup method (automatic monitoring system; UR-1000, UEDA Electronic Works, Tokyo, Japan) without anaesthesia. For the measurement of the vitamin E serum level, blood taken from a tail vein when the rat was killed was centrifuged for $20 \mathrm{~min}$, and vitamin E serum levels were determined by a standard atomic absorption spectrophotometer.

Table 1. Composition of vitamin E-deficient diet

\begin{tabular}{lc}
\hline Cornstarch & $45.5 \mathrm{~g} / 100 \mathrm{~g}$ diet \\
Milk casein & 24.5 \\
Granulated sugar & 10.0 \\
Corn oil & 6.0 \\
Cellulose powder & 5.0 \\
6-Potato starch & 1.0 \\
Vitamin mixture & 1.0 \\
Mineral mixture & 7.0 \\
Vitamin mixture $(\mathrm{mg} / 100 \mathrm{~g}$ diet $)$ & \\
A.D $(500000,100000 \mathrm{IU} / \mathrm{g})$ & 2.40 \\
$\mathrm{~K}_{3}$ & 0.30 \\
$\mathrm{~B}_{1}$ & 1.50 \\
$\mathrm{~B}_{2}$ & 1.56 \\
$\mathrm{~B}_{6}$ & 1.02 \\
$\mathrm{~B}_{12}(0.1 \%)$ & 5.00 \\
Biotin $(2 \%)$ & 0.50 \\
DL-Pantothenic acid calcium & 4.00 \\
PABA & 10.15 \\
Nicotinic acid & 10.15 \\
Inositol & 15.00 \\
Folic acid & 0.20 \\
Choline chloride & 300.00 \\
Cornstarch & 648.22 \\
\hline
\end{tabular}

Before the experiment, the ocular fundus was observed by indirect ophthalmoscopy under ether anaesthesia after dilatation of the pupils with Mydrin-P (tropicamide + phenylephrine).

\section{Corrosion casts and scanning electron microscopy}

After 14 months on the diets, rats in each group were anaesthetised with intraperitoneal sodium pentobarbital. The vascular casts were prepared by our previously described technique. ${ }^{2}$ Briefly, both common carotid arteries were cannulated and the jugular veins were then cut. The vascular system was perfused with heparinised normal saline solution, and freshly prepared Mercox was injected into the cannulated carotid arteries. Then the eyeballs were enucleated, and the ocular tissues were digested with $20 \% \mathrm{KOH}$ for 5 days or longer.

Microdissection was done to expose the retinal vasculature. The casts were rinsed with distilled water and desiccated by freeze-drying, and the dried vascular casts impregnated with osmium overnight, mounted on SEM stubs and coated with gold-palladium. The specimens were examined with a Hitachi S-2360N scanning electron microscope at an accelerating voltage of $10 \mathrm{kV}$. The calibre of the retinal vessels was measured with a caliper micrometer in photographs enlarged to $\times 2$ the original magnification. The capillary branches in a $200 \mu \mathrm{m}$ square were counted in the scanning electron micrographs with the use of Adobe Photoscope.

\section{Light and transmission electon microscopy}

Immediately after the eyes were enucleated under pentobarbital anaesthesia, they were cut in half at the equator and fixed in $4 \%$ glutaraldehyde in $0.05 \mathrm{M}$ cacodylate buffer for $1 \mathrm{~h}$ and postfixed in $1 \%$ osmium tetroxide in veronal acetate buffer for $1 \mathrm{~h}$ after an overnight washing with $0.05 \mathrm{M}$ cacodylate buffer containing $0.44 \mathrm{M}$ sucrose. The fixed materials were dehydrated through a series of ethanols and embedded in Luveak 812.

For light microscopy thin sections were cut with a Porter-Blum MT2 microtome (Sorvall, Newtown, CT) and stained with toluidine blue. For transmission electron microscopy the posterior part of the retina was used. Ultrathin sections were cut with a Porter-Blum MT2 microtome, stained with uranyl acetate and lead citrate, and examined with a Hitachi H300 transmission electron microscope.

\section{Statistical analyses}

Results of determinations are given as mean $\pm \mathrm{SD} ; n$ is the number of experimental animals used or fields examined. The significance of differences was determined by the unpaired Student's $t$-test. Differences were considered significant at $p<0.05$. 

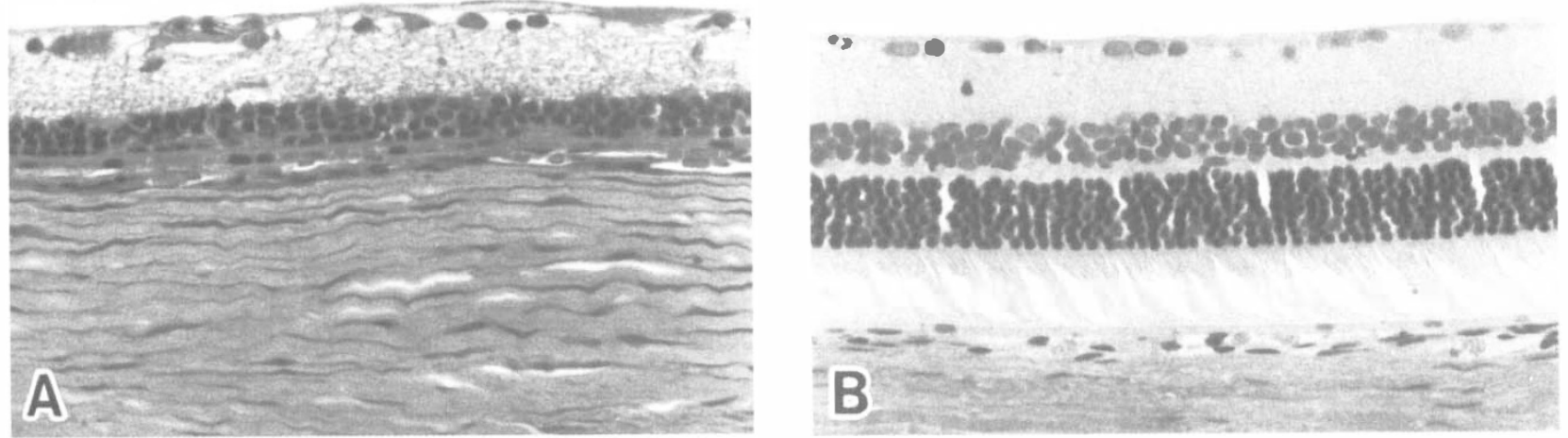

Fig. 1. (A) Light micrograph of a 14-month-old vitamin E-deficient rat showing degenerated retina half as thick as normal retina. A very few photoreceptor cell nuclei are seen. (B) Light micrograph of a 14-month-old normal rat showing full-thickness retina with all layers well defined. Toluidine blue, $\times 200$.
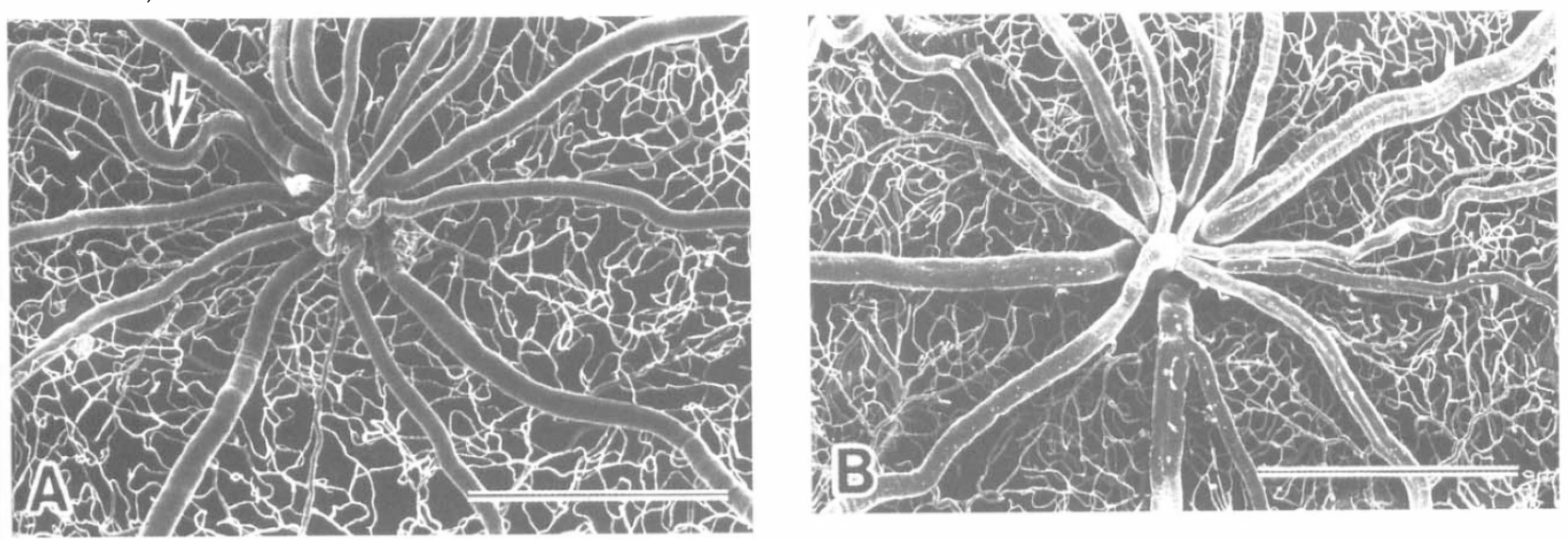

Fig. 2. (A) Scanning electron micrograph of major retinal vessels of a 14-month-old vitamin E-deficient rat. Note the arteriole in the peripapillary region showing tortuosity (arrow). The capillary network is sparse. (B) Normal rat. Scale bar represents $500 \mu \mathrm{m}$.
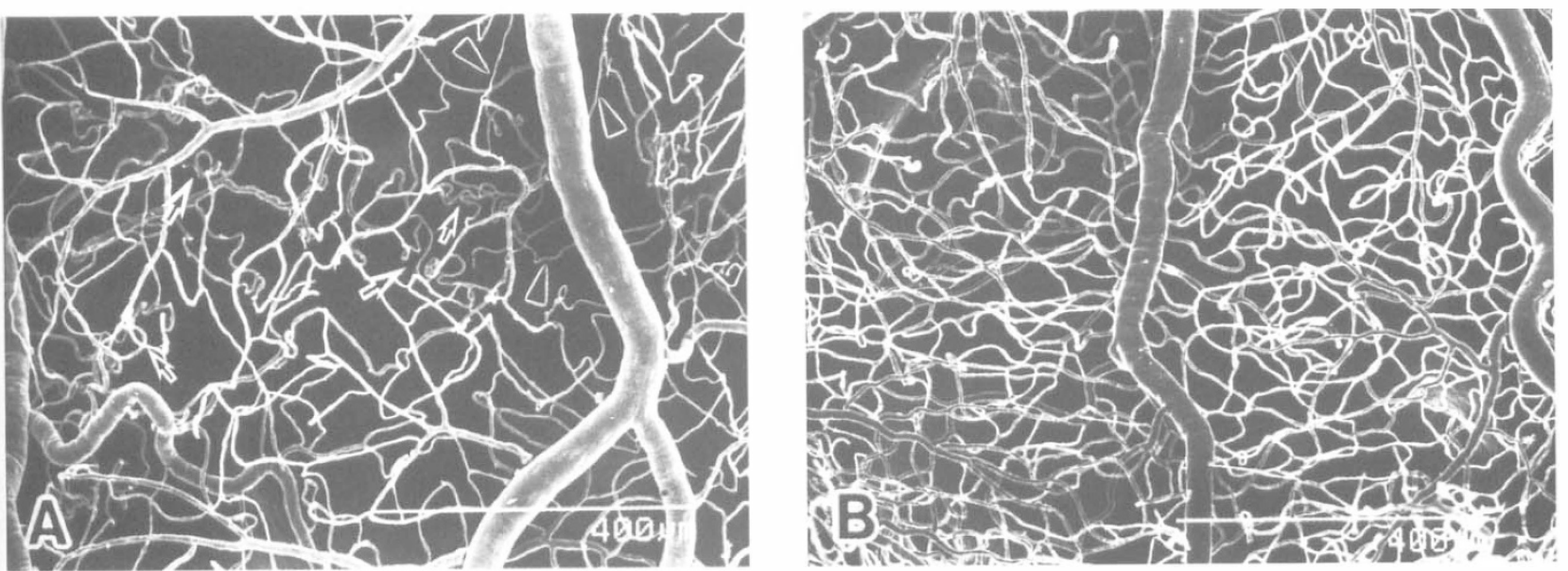

Fig. 3. (A) A magnified view of retinal vessels and capillary networks of a 14-month-old vitamin E-deficient rat showing a sparse capillary network, frequent loop formations (arrows) and local narrowing of capillaries (arrowheads). (B) Normal rat. Scale bar represents $400 \mu \mathrm{m}$.

\section{Results}

\section{Gross characteristics of rats}

In general, the diets produced excellent growth in both groups of rats throughout the experimental period, althouh slight weight loss occurred in the terminal stages in the rats fed the vitamin E-deficient diet. However, there was no statistically significant difference in body weights between the two groups. Weight loss in the rats fed the vitamin E-deficient diet was associated with decreased food intake, which occurred only when plasma tocopherol concentrations fell below $1.5-2.0 \mu \mathrm{l} / \mathrm{ml}$. Rats fed the vitamin E-deficient diet for more than 8 months developed severe myoparesis, limb ataxia and vaginal splinter bleeding (in female rats). 


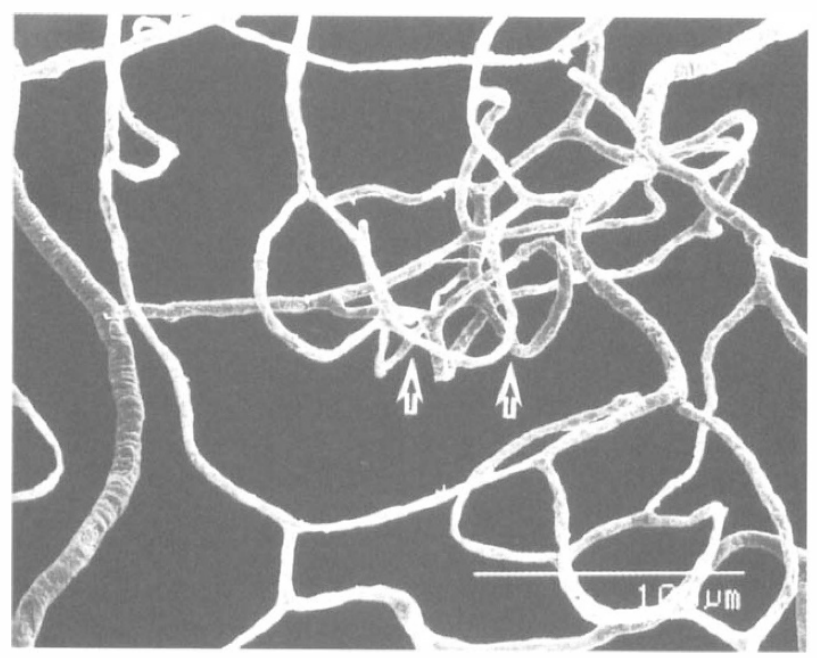

Fig. 4. Scanning electron micrograph of a 14-month-old vitamin Edeficient rat showing sparse thin capillaries. Some of the capillaries are clustered into small tortuous knots (arrows) in the posterior pole of the retina. Scale bar represents $100 \mu \mathrm{m}$.
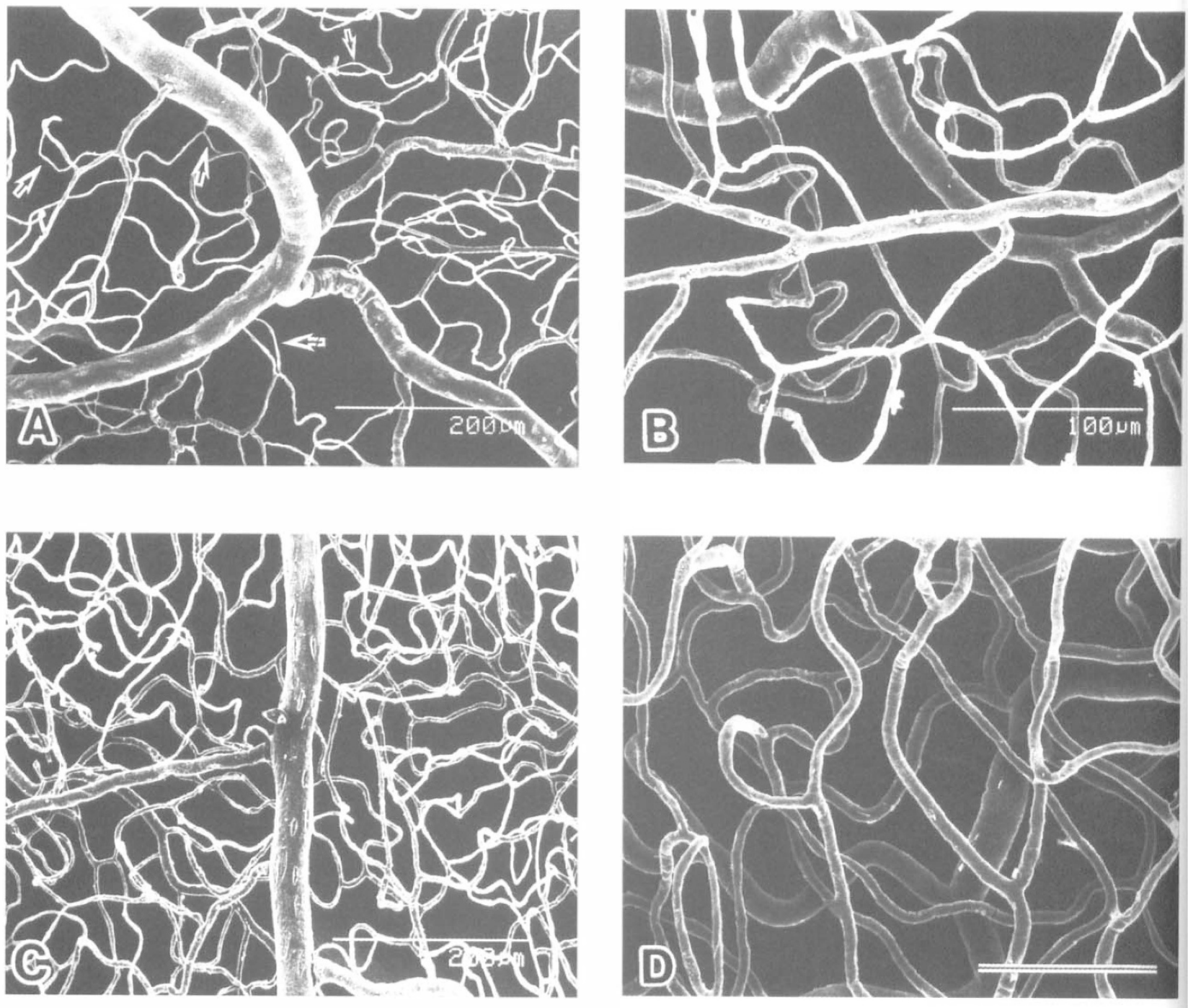

Fig. 5. (A), (B) Anterior (vitreous) views of retinal vessels and capillary network of a 14-month-old vitamin E-deficient rat. In the capillary network there are sparse thin capillaries (arrows) with irregular diameters. Note the two-layered architecture of the capillary network is maintained. (C), (D) In contrast, a normal rat shows a regular and dense capillary network arranged in a two-layered pattern. Scale bars represent $200 \mu \mathrm{m}(A, C)$ and $100 \mu m(B, D)$. 

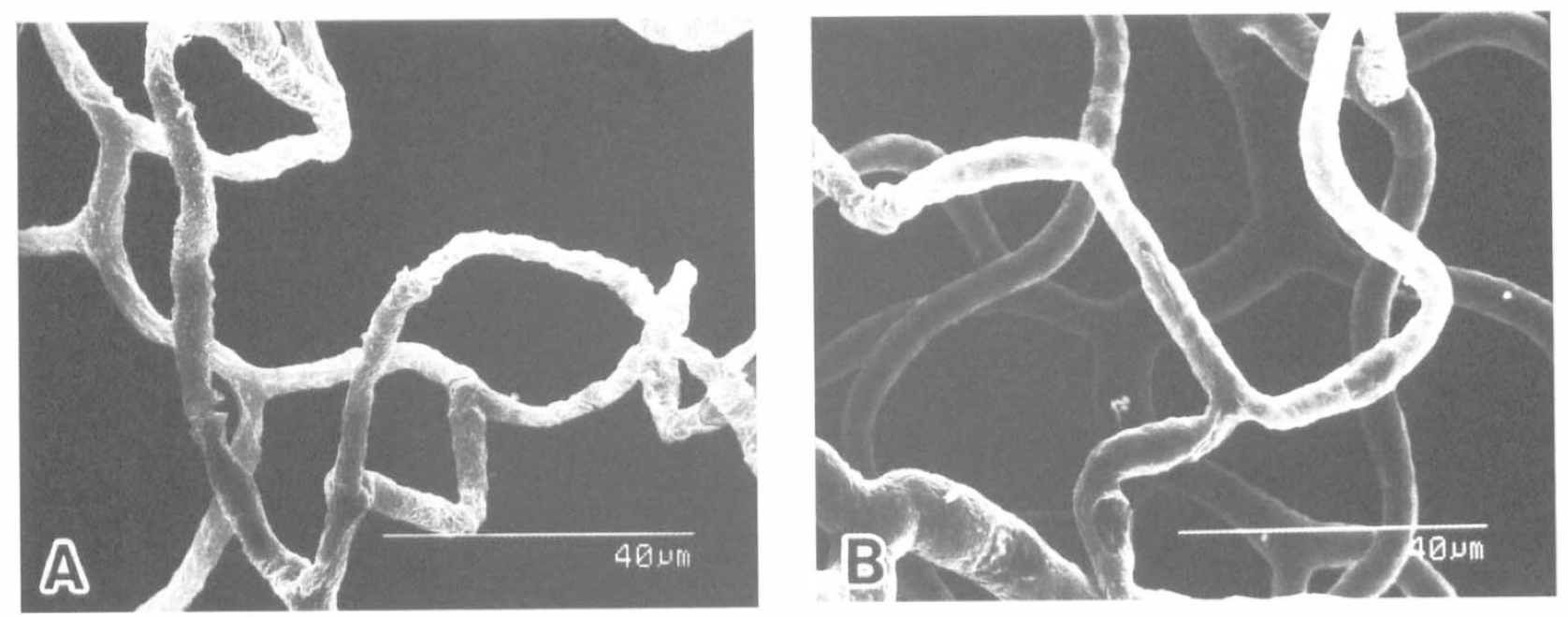

Fig. 6. (A) A magnified view of retinal capillaries of a 14-month-old vitamin E-deficient rat showing fewer endothelial cell nuclear impressions than in a normal rat (B). Scale bar represents $40 \mu \mathrm{m}$.

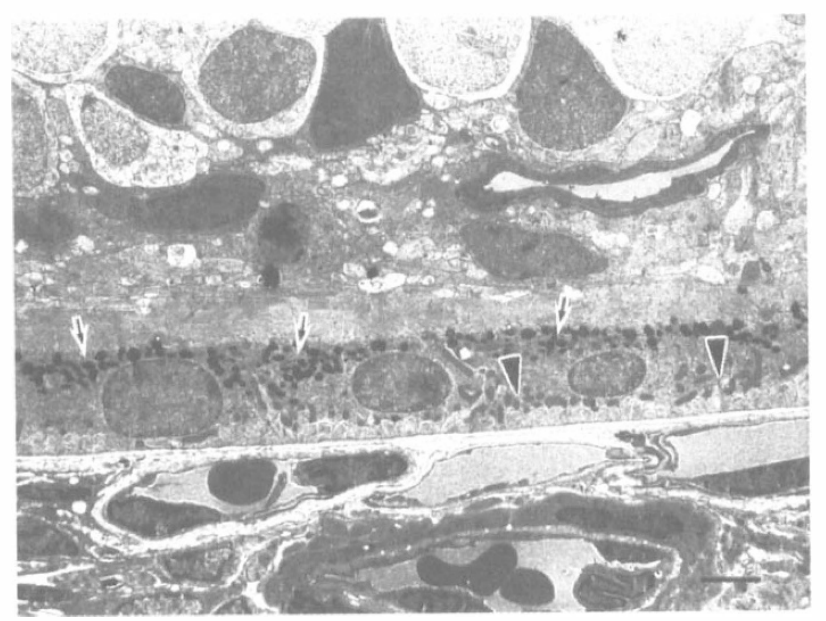

Fig. 7. Transmission electron micrograph of the retina of a rat fed a vitamin E-deficient diet for 14 months. The photoreceptor outer and inner segments and nuclei have completely disappeared. Microvilli of the retinal pigment epithelium (RPE) are in contact with nuclei of the inner nuclear layer cells. The deep retinal capillaries with irregular thickened basement membrane are in direct contact with the RPE. The RPE contains many mitochondria (arrows) and lipofuscin (arrowheads). Scale bar represents $2 \mu \mathrm{m}$.

Slight corneal opacity and keratinisation of focal areas of the cornea were recognised with formation of corneal ulcers and slight lens opacity after 12 months on the vitamin E-deficient diet. No fundus abnormality was detected by indirect ophthalmoscopy. Fourteen months after the start of the experiments, the systolic blood pressure was in the normal range $(115 \pm 8.4 \mathrm{mmHg}$; mean \pm SD) in all normal and vitamin E-deficient rats. The serum level of vitamin $\mathrm{E}$ in rats fed the vitamin E-deficient diet for 12 months was $1.0 \pm 0.49 \mu \mathrm{g} / \mathrm{ml}$, while that of rats fed the normal diet was $13.7 \pm 1.0 \mu \mathrm{g} / \mathrm{ml}$. The difference is statistically significant at $p<0.0001$.

\section{Light microscopic findings}

In 14-month-old vitamin E-deficient rats light microscopy showed thin degenerated retinas half as thick as the normal retinas. The outer plexiform layer was very thin; photoreceptor cell nuclei in the outer nuclear layer decreased to one layer, and the outer and inner segments completely disappeared (Fig. 1A). As a result, photoreceptor cell nuclei were almost in contact with the inner nuclear layer nuclei and the retinal pigment epithelial cells. In contrast, light microscopy of normal rat retinas showed full-thickness and welldefined layers that could be identified easily (Fig. 1B).

\section{Scanning electron microscopic findings}

Scanning electron microscopy (SEM) of vascular casts of the retinas of 14-month-old vitamin E-deficient rats revealed no remarkable micrographic abnormalities in the large retinal vessels other than arterial calibre changes (Fig. 2). The mean calibre of the arteries and veins is shown in Table 2 . The difference in arterial calibre between the vitamin E-deficient and the normal Wistar Kyoto rats is statistically significant $(p<0.022)$. 

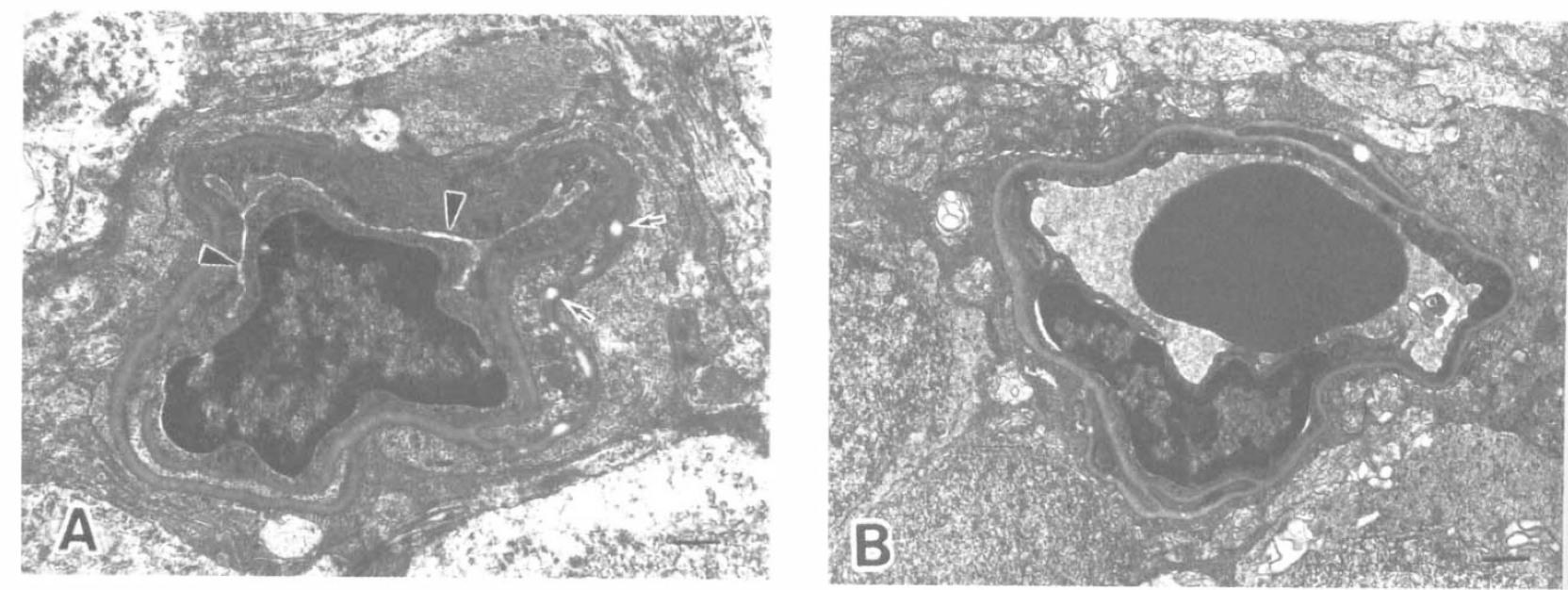

Fig. 8. (A) Transmission electron micrograph of a capillary in the outer plexiform layer of a 13-month-old vitamin E-deficient rat. The lumen of the capillary is very narrow (arrowheads). The capillary has an irregular thickened basement membrane. The endothelium is thickened and has scarce cytoplasmic components with many vesicles. Pericytes contain vacuoles (arrows). (B) Normal rat. Scale bar represents $1 \mu m$.

Some of the arterioles in the peripapillary region showed slight tortuosity (Fig. 2A). However, retinal capillary changes were more evident. The retinal capillaries of the entire retina were decreased in number and scattered. Capillaries showed localised narrowing, caliber irregularity and frequent loop formation (Fig. 3). In the posterior pole of the retina, some capillaries showed clusters of small tortuous knots (Fig. 4). The mean calibre of the retinal capillaries is shown in Table 2 . The difference in capillary diameter between the rats fed the vitamin E-deficient diet and the normal rats is statistically significant $(p<0.0001)$. There were fewer capillary branches $(9.3 \pm 4.6, n=20$ fields examined) in 14-month-old vitamin E-deficient rats than in 14-monthold normal rats $(21.6 \pm 11.2, n=20$ fields examined) in a $200 \mu \mathrm{m}$ square $(p<0.0001)$. Superficial (innermost) capillaries ran parallel to the large retinal vessels. The capillaries were decreased in number but apparently maintained their two layers (Fig. 5A, B). The endothelial nuclear impressions on capillary surfaces were scarcer in vitamin E-deficient rats than in normal rats (Fig. 6A, B). No arteriovenous shunts, crossing defects or microaneurysms were seen. In contrast, none of the normal rats showed any abnormalities (Figs. 2B, 3B, 5C, D).

\section{Transmission electron microscopic findings}

Transmission electron microscopy (TEM) of the retinas of the rats fed the vitamin E-deficient diet for 14 months revealed that photoreceptor outer and inner segments and nuclei had disappeared completely (Fig. 7).
Microvilli of the retinal pigment epithelium (RPE) were in contact with nuclei of the inner nuclear layer. The outer plexiform layer-like structures remained interrupted between the RPE and the inner nuclear layer. The RPE contained many mitochondria, lipofuscin (Fig. 7) and retinal capillaries. The retinal capillaries in the inner and outer plexiform layers and in the RPE had narrow lumens (Fig. 8A). The endothelial cells were thickened and had scarce cytoplasmic components with vacuoles and irregularly thickened basement membranes. The capillary pericytes contained vacuoles (Fig. 8A).

\section{Discussion}

The architecture of the retinal vasculature in the rat is composed of four or five large arteries and veins, arterioles and venules, and capillaries which are distributed in a two-layer pattern of the network between the nerve fibre layer and the outer plexiform layer. When the retina is affected with focal or total degeneration, defects or total thinning, the architecture of the vascular changes is not clear. One main question was whether the two-layer pattern of the capillary network is maintained in the degenerated retina. We chose vitamin E-deficient rat retinas because in vitamin $\mathrm{E}$ deficiency the retina is degenerated and thin ${ }^{4,5}$ and the retinal vessels are damaged. ${ }^{3}$ Corrosion casts and SEM and TEM were used for this retinal vascular study because corrosion casts

Table 2. Mean calibre of retinal vessels and capillaries

\begin{tabular}{lccc}
\hline Rat & Artery & Vein & Capillary \\
\hline Vitamin E-deficient & $36.73 \pm 1.04 \mu \mathrm{m}$ & $47.07 \pm 1.47 \mu \mathrm{m}$ & $3.98 \pm 0.15 \mu \mathrm{m}$ \\
$(14$ months old $)$ & $(n=46)$ & $(n=46)$ & $(n=60)$ \\
Normal & $38.56 \pm 4.24 \mu \mathrm{m}$ & $48.75 \pm 6.21 \mu \mathrm{m}$ & $8.52 \pm 0.21 \mu \mathrm{m}$ \\
$(14$ months old $)$ & $(n=46)$ & $(n=46)$ & $(n=60)$ \\
& $p<0.022$ & $p=0.147$ & $p<0.0001$ \\
\hline
\end{tabular}

$n$, number of fields examined. 
and SEM provide a three-dimensional view of the retinal vascular architecture ${ }^{1,2}$ and TEM shows detailed changes of the vessels.

In the present study, the vitamin E-deficient rats showed a loss of photoreceptor outer and inner segments, of nuclei and of the outer plexiform layer. The retina was half as thick as the normal rat retina. These findings have already been reported ${ }^{6,7}$ and have been considered to indicate the following: vitamin $\mathrm{E}$ is a fatsoluble vitamin which acts as a lipid antioxidant and has a protective role in membrane-bound enzyme functions through the inhibition of peroxidation and radical formation. ${ }^{8}$ Vitamin $\mathrm{E}$ is normally present in high concentrations in the membranes of rod outer segments of the retina, ${ }^{9}$ an area which is particularly susceptible to peroxidation. This is because the outer segment contains high concentrations of polyunsaturated fatty acids ${ }^{10}$ and receives a plentiful supply of oxygen, and light is known to enhance peroxidation. ${ }^{11} \mathrm{~A}$ deficiency of vitamin $\mathrm{E}$ is therefore likely to accenuate peroxidative damage to the retina and to increase oxidative destruction of vitamin $\mathrm{E}$.

The retinas of rats fed a vitamin E-deficient diet for 14 months were very thin because of disappearance of the photoreceptor cells and outer plexiform layer. Normally the retinal vessels are present from the outer plexiform layer to the nerve fibre layer. The photoreceptor cells, i.e. the retina from the outer nuclear layer to the outer segments, have no vasculature and are supplied with oxygen and nutrients from the vessels in the outer plexiform layer and choriocapillaries through the RPE. It is very interesting to consider what happens to the blood vessel architecture in the retina when blood vessels lose their target of blood supply in retinal degeneration such that seen in vitamin E-deficient rat retinas. The present study showed that the two-layer capillary network was maintained: the deep retinal capillary bed was brought into close proximity with the RPE as a consequence of degeneration of the outer neuroretinal layers. The sparsity of capillaries is understandable because capillaries themselves degenerate in vitamin E deficiency and because retinal thinning and degeneration and loss of retinal structures result in a reduced need for oxygen and nutrient supplies. The vascular changes in the vitamin E-deficient rat retina were similar to that previously reported in rats with spontaneous diabetes (WBN/Kob) with hereditary retinal degeneration: ${ }^{12}$ a two-layer capillary network is maintained in the thin degenerated retina. Although the cause of retinal degeneration may be different in each animal model, gradual disappearance of a retinal capillary network is considered to be one of the common pathologies in degenerated retina.

Since vessel distribution may be determined by the blood requirements of degenerated tissue, it is mysterious that the deep capillaries persist. These findings are surprising because retinal thickness and vascularity are normally positively correlated. Davson ${ }^{13}$ and Bellhorn ${ }^{14}$ believe that vessel morphology is determined by the requirements of the tissue it subserves. Thus, if a vessel is placed in an unusual environment, the morphology of that vessel may change. We previously reported that the superficial capillary network in the rat retina comes essentially from arterioles, while deep layer capillaries of the network are more regularly and densely arranged and are connected mainly with venules. ${ }^{2}$ This anatomical structure may explain why the deep capillaries of the network may not act as feeders; even if they have a role in feeding the RPE, it would be a small role because the RPE is nourished by choriocapillaries. Since the superficial capillaries are distributed to the whole retina, the deep capillaries are necessary for the retina pathway. This might be why the two-layer network is maintained.

On the other hand, retinal vessels themselves were affected by vitamin E deficiency: decrease of capillaries, capillary membrane thickening, capillary lumen narrowing due to thickened endothelium, pericyte damage, irregular calibre of capillaries and tortuous large vessels. Most of these findings have also been reported. ${ }^{3}$ Since vascular cells are damaged by free radicals and lipid peroxides generated by oxidation, vitamin E may act on the vascular wall to prevent cellular damage and maintain function. ${ }^{15}$

The effects of vitamin E deficiency on capillaries vary from tissue to tissue. We were interested in what happens to the retinal vascular architecture in the diffusely degenerated thin retina. The time course of degeneration related to the vascular anomalies is particularly important. There are few detailed morphological and quantitative studies on the alterations in retinal vessels in retinal degeneration. ${ }^{16,17}$ Although these studies demonstrated changes in the retinal vascular architecture with the progress of retinal degeneration, they did not describe the threedimensional architecture of the retinal capillary network.

The present study has shown that vitamin $\mathrm{E}$ deficiency causes degeneration throughout the retina and retinal thinning, a decrease in the number of retinal capillaries and irregularity of retinal caliber. In spite of these effects of vitamin E deficiency, the two-layer pattern of the capillary network was maintained. This means that the basic pattern of retinal vascular architecture may be maintained in retinal degeneration despite local changes such as the decrease in the number of capillaries and changes in the entire retina with thickening or thinning of layers.

\section{References}

1. Pannarale L, Onori P, Ripani M, Gaudio E. Retinal microcirculation as revealed by SEM corrosion casts in the rat. Eur J Ophthalmol 1991;1:96-102.

2. Bhutto IA, Amemiya T. Corrosion cast demonstration of retinal vasculature of normal Wistar Kyoto rats. Acta Anat 1995;153:290-300.

3. Amemiya T. Effects of vitamin $E$ and selenium deficiencies on rat capillaries. Int J Vit Nutr Res 1989;59:122-6.

4. Riis RC, Sheffy BE, Loew K, Kern TJ, Smith JS. Vitamin E deficiency retinopathy in dogs. Am J Vet Res 1981;42:74-86.

5. Hayes KC. Retinal degeneration in monkeys induced by deficiencies of vitamin E or A. Invest Ophthalmol 1974;13:499-510. 
6. Robison WG Jr, Kuwabara T, Bieri JG. Deficiencies of vitamins $\mathrm{E}$ and $\mathrm{A}$ in the rat, retinal damage and lipofuscin accumulation. Invest Ophthalmol Vis Sci 1980;19:1030-7.

7. Amemiya T. Effects of vitamin E administration on photoreceptor outer segment and retinal pigment epithelium of vitamin E deficient rats. Int J Vit Nutr Res 1981;51:114-8.

8. Runge P, Muller DPR, McAllister J, Calver D, Llyod JK, Taylor D. Oral vitamin E supplements can prevent the retinopathy of abetalipoproteinaemia. Br J Ophthalmol 1986;70:166-73.

9. Dilley RA, McConnell DG. Alpha-tocopherol in the retina outer segment of bovine eyes. J Membr Biol 1970;2:317-22.

10. Daemen FJM. Vertebrate rod outer segment membranes. Biochim Biophys Acta 1973;300:255-65.

11. Feeney L, Berman ER. Oxygen toxicity: membrane damage by free radicals. Invest Ophthalmol Vis Sci 1976;15:789-91.
12. Bhutto IA, Miyamura N, Amemiya T. Vascular architecture of degenerated retina in WBN/Kob rats: corrosion cast and scanning electron microscopic study. Ophthalmic Res 1999;31:367-77.

13. Davson $\mathrm{H}$. The Bowman lecture. The little brain. Trans Ophthalmol Soc UK 1979;99:21-37.

14. Bellhorn RW. Control of blood vessel development. Trans Ophthalmol Soc UK 1980;100:328-31.

15. Tappel AL. Vitamin E and free radical peroxidation of lipids. Ann NY Acad Sci 1972;203:12-28.

16. Methes MT, Bok D. Blood vascular abnormalities in the degenerative mouse retina (C57BL/6J-rd). Invest Ophthalmol Vis Sci 1984;25:364-9.

17. Blanks JC, Johnson LV. Vascular atrophy in the retinal degeneration rd mouse. J Comp Neurol 1986;254:543-53. 